\title{
Análisis aerodinámico en CFD de los alerones en automóviles convencionales
}

\section{Aerodynamic analysis in CFD of spoilers in conventional cars}

\author{
HORTELANO-CAPETILLO, Juan Gregorio†*, MARTÍNEZ-VÁZQUEZ, J. Merced y RODRIGUEZ- \\ ORTIZ, Gabriel
}

Universidad Politécnica de Juventino Rosas, México.

ID $1^{\mathrm{er}}$ Autor: Juan Gregorio, Hortelano-Capetillo / ORC ID: 0000-0002-3702-4853

ID $1^{\mathrm{er}}$ Coautor: J. Merced, Martínez-Vázquez / ORC ID: 0000-0002-6230-3846

ID $2^{\text {do }}$ Coautor: Gabriel, Rodriguez-Ortiz / ORC ID: 0000-0002-3615-1973

DOI: $10.35429 / J I E .2020 .11 .4 .1 .12$

Recibido Enero 15, 2020; Aceptado Marzo 30, 2020

\section{Resumen}

En el presente estudio se realizaron pruebas aerodinámicas mediante el uso del software Solidworks Flow Simulation (CFD) en 2 diseños diferentes de automóviles convencionales, implementando un aleron en la parte trasera del auto para disminuir los coeficientes de arrastre y de sustentación que se producen mediante el movimiento del aire independientemente del diseño en la parte trasera del auto y mejorar la aerodinámica. Analizando los resultados se obtiene que se mejora la aerodinámica del automóvil cuando se le pone un alerón para reducir las fuerzas de arrastre y sustentación.

Coeficiente de arrastre, Coeficiente de sustentación, Aerodinámica

\begin{abstract}
In the present study aerodynamic tests were performed by using Solidworks Flow Simulation software (CFD) in 2 different designs of conventional cars, implementing a spoiler in the back of the car to reduce the drag and lift coefficients that occur through the movement of the air regardless of the design in the back of the car and improve aerodynamics. Analyzing the results it is obtained that the aerodynamics of the car is improved when a spoiler is placed to reduce the drag and lift forces.
\end{abstract}

Drag coefficient, Lift coefficient, aerodynamics

Citación: HORTELANO-CAPETILLO, Juan Gregorio, MARTÍNEZ-VÁZQUEZ, J. Merced y RODRIGUEZ-ORTIZ, Gabriel. Análisis aerodinámico en CFD de los alerones en automóviles convencionales. Revista de Ingeniería Industrial. 2020. 4-11:1-12.

*Correspondencia al Autor (Correo Electrónico:jhortelano_ptc@upjr.edu.mx)

$\dagger$ Investigador contribuyendo como primer autor. 


\section{Introducción}

La aerodinámica es el estudio del aire en movimiento sobre un sólido determinando las presiones y las fuerzas. Se originan 3 tipos de fueras sobre el automóvil: fuerza de arrastre que dificulta el movimiento del vehículo, Fuerza de sustentación que es la fuerza vertical hacia arriba o abajo apretando el vehículo contra el suelo y Fuerza lateral [1]. Los problemas aerodinámicos son complejos, ya que son problemas de una alta complejidad, haciéndolos imposibles de resolver mediante los métodos analíticos; por lo que la solución a estos problemas requiere de otros métodos, que nos da resultados aproximados. Principalmente existen dos métodos de resolución de problemas aerodinámicos:

- $\quad$ Túnel de viento. - Técnicas CFD (Computacional Fluid
Dynamics).

Décadas después, extensivos análisis en túnel de viento realizados por ingenieros de Chrysler demostraron que los automóviles estaban diseñados "literalmente a la inversa". Los pésimos diseños luchaban contra el viento lo que tenía como resultado combustible malgastado y bajas velocidades. Todos estos hechos se demostraron en el modelo de Chrysler denominado DeSoto de 1933 que fue diseñado para ser conducido en el sentido inverso. Dichas pruebas dieron como resultado que este modelo aportó más kilómetros por litro y velocidades máximas mayores [2].

Una fuerza aerodinámica de sustentación en la parte delantera del coche fue percibida por Daimler-Benz durante un recorrido que impartió récord en la autopista alemana en el periodo comprendido entre los años 1936 y 1939. A velocidades mayores a $200 \mathrm{mph}$ Carraciola y Lang observaron que la parte delantera del coche parecía elevarse produciendo una pérdida en la sensibilidad de la dirección. En una carrera en la que se alcanzó una velocidad aproximada de 250 mph, Caracciola reportó una completa pérdida de control de la dirección, así como los observadores tuvieron la impresión de que las llantas delanteras se levantaron del suelo [3].
El implemento de un alerón en la parte trasera del automóvil empezó Michael May, ingeniero suizo y piloto de carreras aficionado, fue el primero en experimentar con un ala invertida montada sobre la cabina de su Porsche 550 Spyder en el año 1956. En los años 60 con Jim Hall (1965) en su auto Chaparral 2E implemento el alerón. Posteriormente en 1969 en la NASCAR con el Dodge Charger Daytona y su gemelo el Plymouth Superbird debido a que el reglamento de entonces era muy estricto en cuanto a los motores, por lo que los directivos del grupo Chrysler, al que pertenecían Dodge y Plymouth, vieron en la aerodinámica una posible oportunidad de superar a los rivales. Tras innumerables pruebas en el túnel del viento de la empresa aeronáutica Lockheed, instalaron un inmenso alerón trasero, que a pesar de lo que algunos creen, es muy eficaz debido a su elevada posición [4].

La Figura 1 muestra la evolución de los alerones a través de los años para reducir y optimizar la resistencia que ofrece el automóvil al aire y disminuir las fuerzas de arrastre y sustentación. R.B. Sharma et al., [5] mencionan que el proceso del diseño de un automóvil, la aerodinámica debe ser considerada muy seriamente, un auto puede ser aceptable si su forma reduce el coeficiente de arrastre.
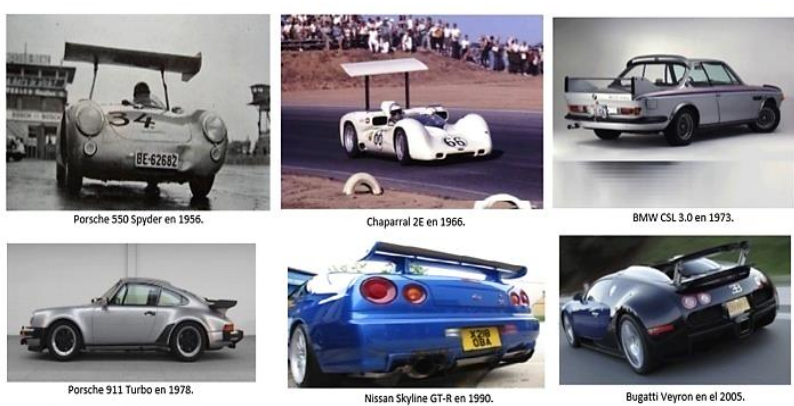

Figura 1 Alerones en diferentes diseños de automóviles Fuente: [2,3,4]

Carr G. W. [6] descubrió que las fuerzas aerodinámicas son un factor importante en el diseño de los automóviles en competición, puede ser rápido o lento dependiendo de su forma usando la misma potencia en el motor. Goetz [7] midió las distribuciones de las presiones alrededor de un automóvil dentro de un túnel de viento. Las superficies sustentadoras (alerones) diseñadas por Liebeck [8] fueron usados por automóviles de competencia para generar el auto más rápido y aerodinámico. 
R.H. Barnard et al., [9] realizaron pruebas aerodinámicas en un túnel de viento de un auto en competición marca Tiga GC83 a escala de $1 / 8$, obteniendo como resultados los valores de los coeficientes de arrastre y sustentación.

Los métodos aerodinámicos fueron avanzando tanto la parte experimental como la parte de simulación numérica. Chien-Hsiung Tsai et al., [10] realizaron pruebas aerodinámicas en un automóvil con 5 diferentes diseños de alerones obteniendo como resultado la disminución del coeficiente de arrastre con el mejor diseño del alerón. Xingjun HU et al., [11] realizaron simulaciones en CFD de un auto Sedan para analizar el difusor a diferentes ángulos de corte $\left(0^{\circ}, 3^{\circ}, 6^{\circ}, 9.8^{\circ}\right.$ y $\left.12^{\circ}\right)$ que se encuentra ubicado en la parte trasera del auto, para determinar los coeficientes de arrastre y sustentación, observó que con un ángulo de corte de $6^{\circ}$, los coeficientes son menores en comparación con los resultados con los demás ángulos de corte.

El mismo análisis en el difusor usando CFD lo realizaron S. M. Rakibul Hassan et al., [12] pero con ángulos de corte $0^{\circ}, 2.5^{\circ}, 5^{\circ}, 10^{\circ}$ y $12.5^{\circ} \mathrm{y}$ analizaron que con un ángulo de corte de $12.5^{\circ}$ se obtiene el menor coeficiente de arrastre en comparación con los demás ángulos de corte. Rubel Chandra Das et al., [13] realizaron simulaciones en CFD de un automóvil con su alerón en la parte trasera variando los ángulos de ataque del aire de $2^{\circ}, 4^{\circ}, 6^{\circ}, 8^{\circ}, 10^{\circ}$ y $12^{\circ}$ para optimizar los coeficientes de arrastre y sustentación.

Obteniendo como resultado que con un ángulo de inclinación en el alerón de $4^{\circ}$ y $12^{\circ}$ se obtienen los menores coeficientes. Krzysztof Kurec et al., [14] realizaron pruebas en CFD y experimentales de un auto normal con un alerón en la parte trasera, variando los ángulos de ataque desde $0^{\circ}$ hasta $55^{\circ}$, probando diferentes modelos de turbulencia, observaron que el coeficiente de arrastre aumenta conforme el ángulo del alerón va aumentando los grados de inclinación. Algunos investigadores Gilhaus et al., [15], J. R. Callister et al., [16], F. R. Bailey et al., [17], H. Taeyoung et al., [18] usaron técnicas en CFD para obtener resultados numéricos de los automóviles.
Los autores (Cooper [19] y Sovran [20]) comentan que reduciendo un $10 \%$ de la fuerza de arrastre se logra reducir el consumo de combustible alrededor de 3-4\%. Katz [21] y Regert et al., [22] probaron que la fuerza de arrastre y la distribución de presión alrededor del vehículo depende del diseño y no de la fricción de la superficie. La reducción del área de la parte trasera de los vehículos ha sido estudiada por décadas para reducir el arrastre que se genera por la separación del aire (Wong et al., [23], Lee et al., [24] y Mariotti et al., [25]), Algunos autores Beaudoin et al., [26], Fourrié et al., [27], Hanfeng et al., [28], Grandemange et al., [29] y Urquhart et al., [30] investigaron que el mejor dispositivo para mejorar la aerodinámica esta dado por un alerón, deflector o aletas.

\section{Modelo matemático y numérico}

El uso del software CFD no es una disciplina nueva, gracias a las grandes mejoras en el desarrollo de las computadoras han hecho de la simulación numérica una herramienta de suma utilidad en la ingeniería. En este trabajo se realizaron varios ensayos en CFD para reproducir el comportamiento aerodinámico para los diferentes modelos con el implemento de un alerón. La Figura 2 muestra los pasos para resolver un problema utilizando CFD.

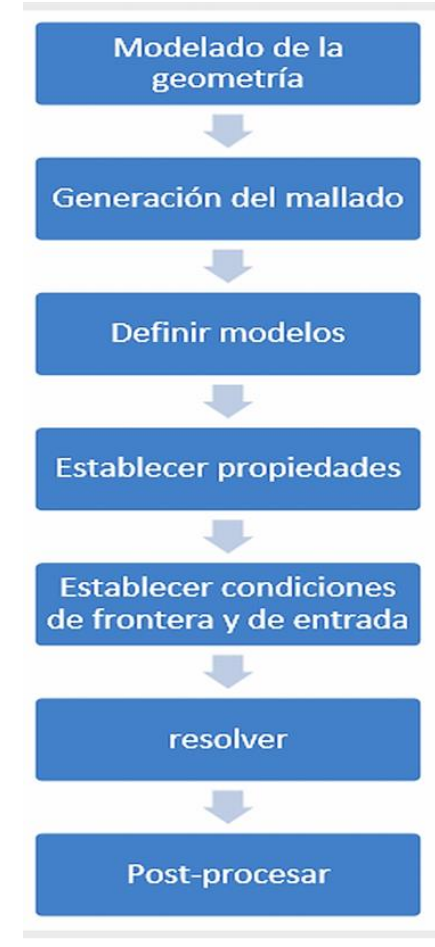

Figura 2 Pasos para la simulación en CFD Fuente: elaboración propia 
Para un análisis aerodinámico externo de un cuerpo se deben tomar en cuenta algunos coeficientes entre los cuales se encuentran el coeficiente de arrastre y el coeficiente de sustentación. Los modelos de automóviles fueron diseñados en Solidworks, posteriormente se realizaron las simulaciones aerodinámicas usando el complemento Flow Simulation.

\begin{abstract}
Se diseñaron 2 automóviles convencionales para compararlos aerodinámicamente entre sí, posteriormente se diseño un alerón para colocarlo en la parte trasera de los modelos variando la altura de 10 $\mathrm{cm}$ hasta $45 \mathrm{~cm}$.
\end{abstract}

\section{Diseño 1}

Se diseñó el primer modelo con dimensiones 1.8 $\mathrm{m}$ de alto, $2.6 \mathrm{~m}$ de ancho y $4.14 \mathrm{~m}$ de largo. Como se muestra en la Figura 3.

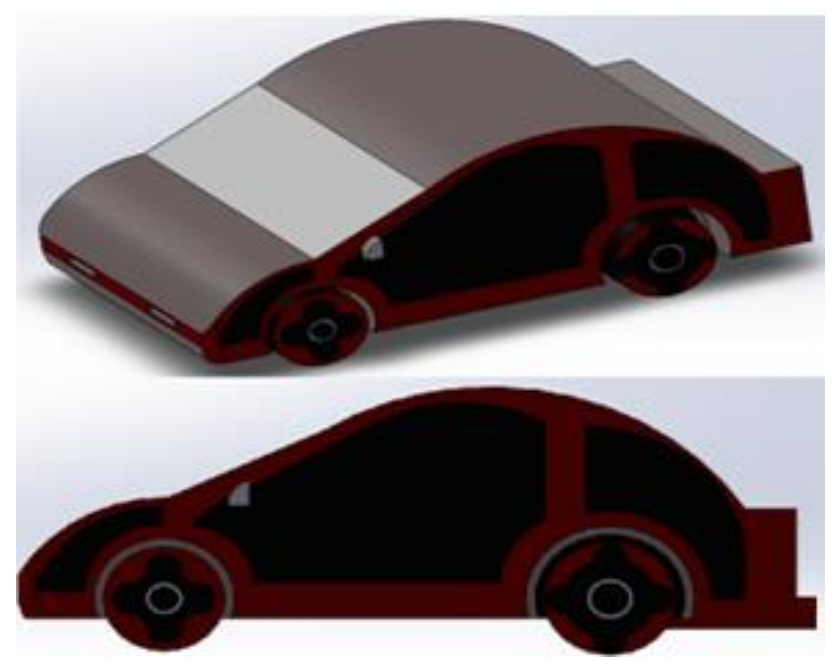

Figura 3 Diseño 1. Diseño de un automóvil convencional Fuente: elaboración propia [Solidworks]

\section{Diseño 2}

Se diseñó el segundo modelo con dimensiones $1.8 \mathrm{~m}$ de alto, $2.6 \mathrm{~m}$ de ancho, las mismas dimensiones que el diseño 1, pero con una longitud de $4 \mathrm{~m}$ de largo. Como se muestra en la Figura 4.

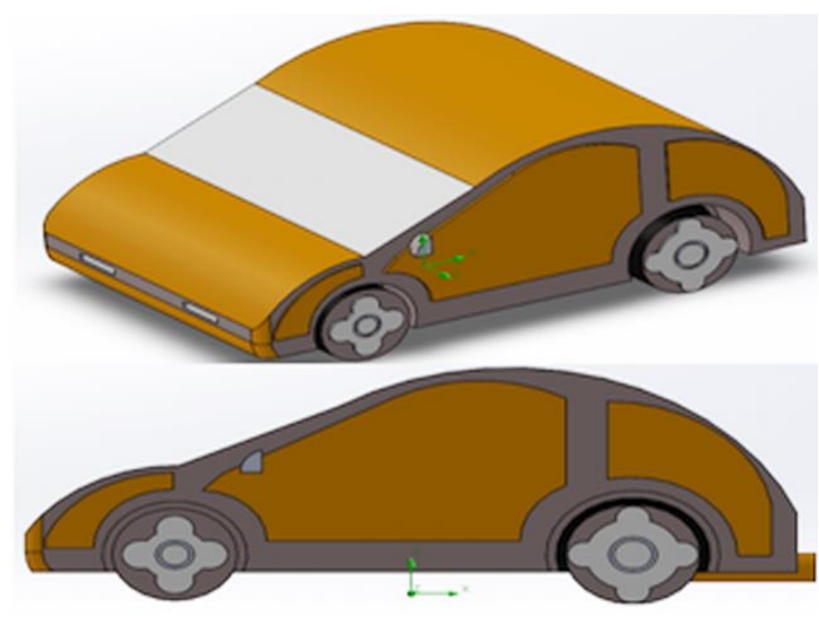

Figura 4 Diseño 2. Diseño de un auto normal Fuente: elaboración propia [Solidworks]

\section{Diseño 1/Alerón 1}

Se le implemento un alerón en la parte trasera del automóvil mostrado en la Figura 5, el alerón está a una altura de $10 \mathrm{~cm}$, el alerón tiene un ancho de $30 \mathrm{~cm}$ con un espesor de $4 \mathrm{~cm}$.

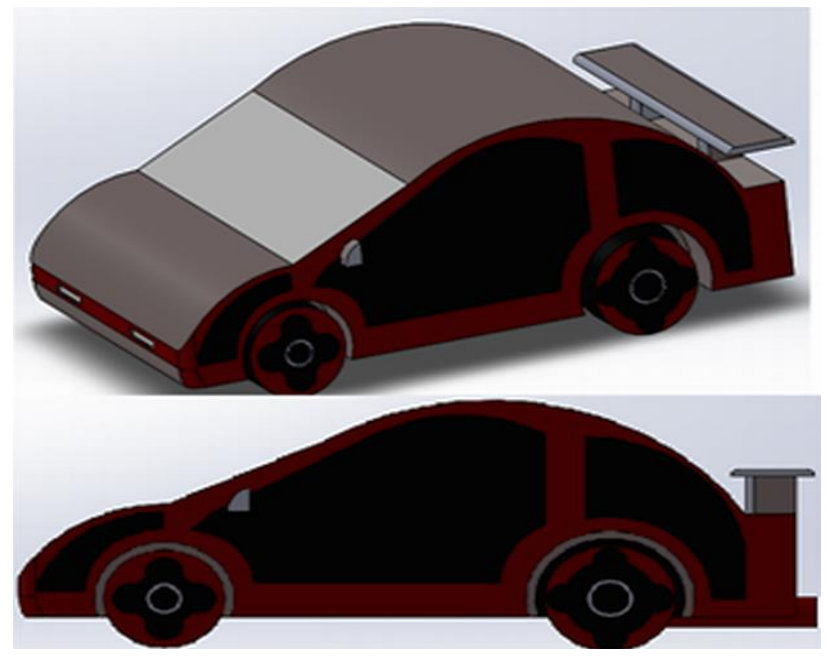

Figura 5 Diseño 1/Alerón 1. Aleron implementado en la parte trasera del auto de $10 \mathrm{~cm}$ de altura Fuente: elaboración propia [Solidworks]

\section{Diseño 1/Alerón 2}

Se le implemento el mismo alerón, pero con una altura de $45 \mathrm{~cm}$, como se observa en la Figura 6. 


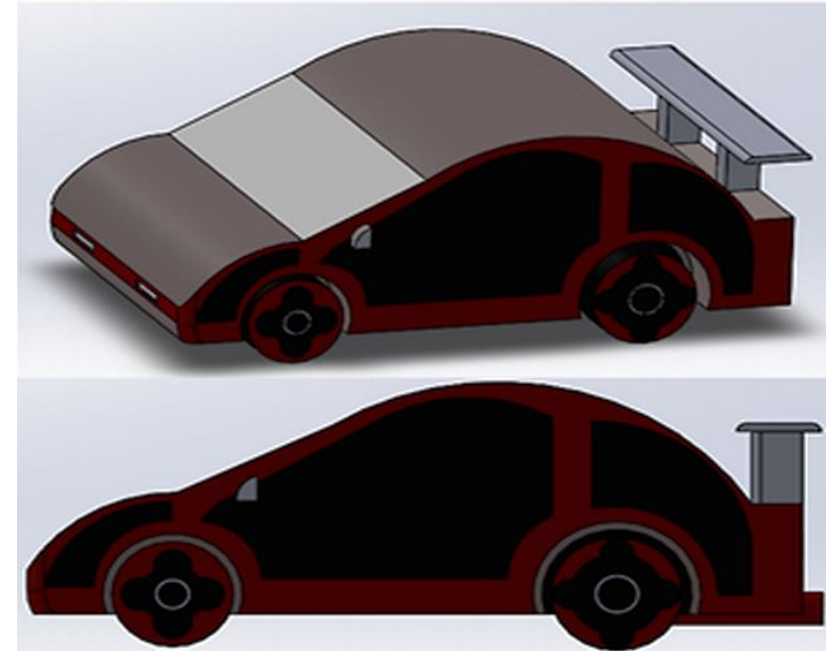

Figura 6 Diseño 1/Alerón 2. Aleron implementado en la parte trasera del auto de $45 \mathrm{~cm}$ de altura

Fuente: elaboración propia [Solidworks]

\section{Diseño 2/Alerón 1}

A este modelo se le implemento un alerón en la parte trasera del automóvil mostrado en la Figura 7 , el alerón tiene una altura de $10 \mathrm{~cm}$.

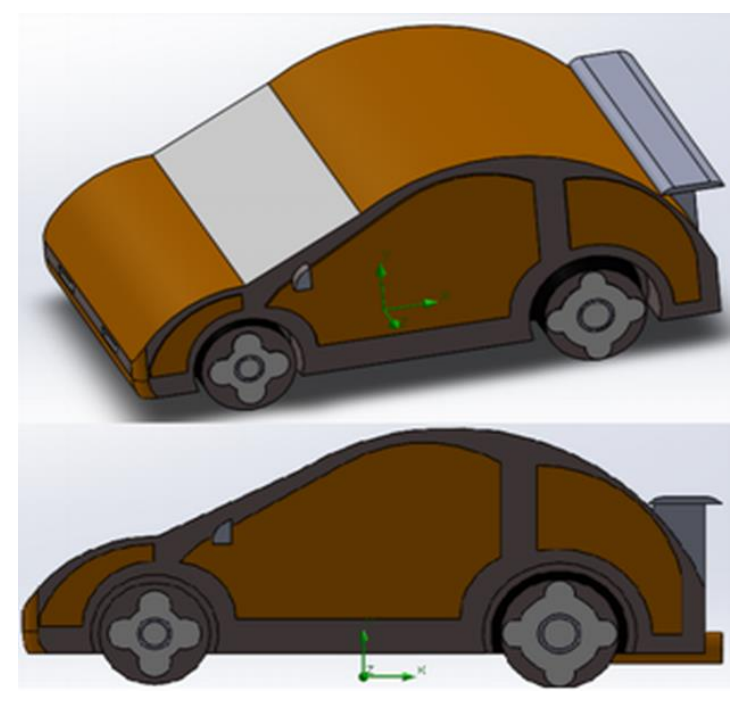

Figura 7 Diseño 2/Alerón 1

Fuente: elaboración propia [Solidworks]

\section{Diseño 2/Alerón 2}

Se le implemento un alerón en la parte trasera del automóvil mostrado en la Figura 8, el alerón tiene una altura de $45 \mathrm{~cm}$.

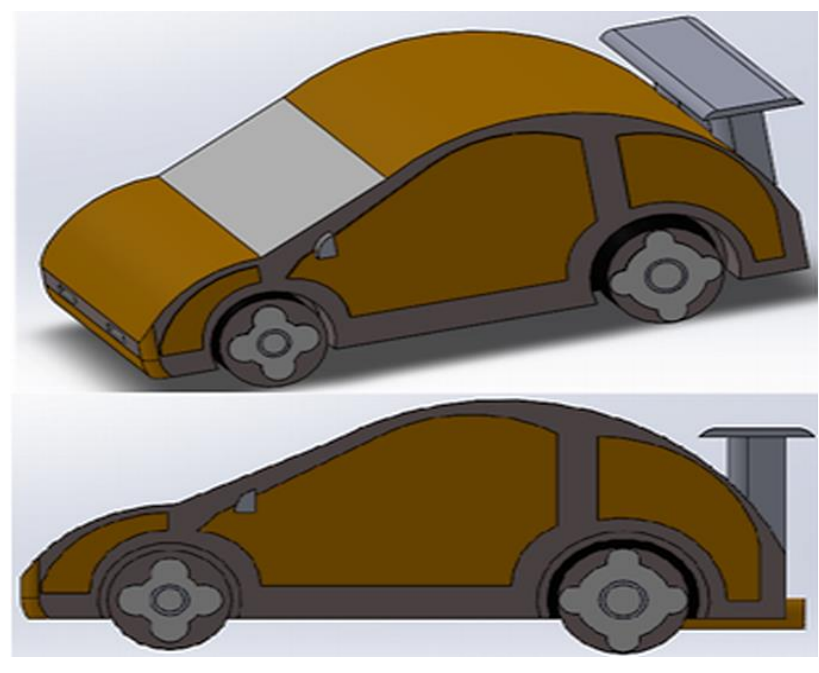

Figura 8 Diseño 2/Alerón 2

Fuente: elaboración propia [Solidworks]

Para este caso de estudio se usa un mallado volumétrico que se forma utilizando celdas hexaédricas como se muestra en la Figura 9, generalmente es usado para la aerodinámica externa por su capacidad para definir correctamente la zona donde se forma la estela debido a la separación de la capa límite volviendo al fluido turbulento.

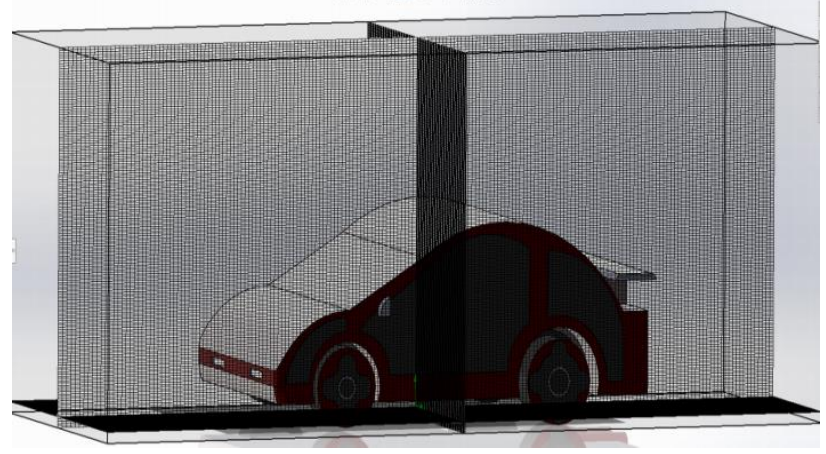

Figura 9 Volumen de control con 5313028 celdas en el mallado

Fuente: elaboración propia [Solidworks]

El estudio aerodinámico que presenta el flujo desarrollado alrededor del vehículo requiere de una presentación matemática, que a su vez se transforma en un algoritmo para su solución. Esta presentación matemática se resume en un conjunto de ecuaciones de conservación de masa, cantidad de movimiento y el modelo de turbulencia $k-\varepsilon$.

La ecuación de continuidad está representada por [31]:

$\frac{\partial u}{\partial x}+\frac{\partial v}{\partial y}+\frac{\partial w}{\partial z}=0$

Las ecuaciones de Navier Stokes se expresan de la siguiente forma [1]:

HORTELANO-CAPETILLO, Juan Gregorio, MARTÍNEZVÁZQUEZ, J. Merced y RODRIGUEZ-ORTIZ, Gabriel. Análisis aerodinámico en CFD de los alerones en automóviles convencionales. Revista de Ingeniería Industrial. 2020 
$\rho\left(\frac{\partial u}{\partial t}+u \frac{\partial u}{\partial x}+v \frac{\partial u}{\partial y}+w \frac{\partial u}{\partial z}\right)=\rho g_{x}-\frac{\partial P}{\partial x}+\mu\left(\frac{\partial^{2} u}{\partial x^{2}}+\frac{\partial^{2} u}{\partial y^{2}}+\frac{\partial^{2} u}{\partial z^{2}}\right)$

$\rho\left(\frac{\partial v}{\partial t}+u \frac{\partial v}{\partial x}+v \frac{\partial v}{\partial y}+w \frac{\partial v}{\partial z}\right)=\rho g_{y}-\frac{\partial P}{\partial y}+\mu\left(\frac{\partial^{2} v}{\partial x^{2}}+\frac{\partial^{2} v}{\partial y^{2}}+\frac{\partial^{2} v}{\partial z^{2}}\right)$

$\rho\left(\frac{\partial w}{\partial t}+u \frac{\partial w}{\partial x}+v \frac{\partial w}{\partial y}+w \frac{\partial w}{\partial z}\right)=\rho g_{z}-\frac{\partial P}{\partial z}+\mu\left(\frac{\partial^{2} w}{\partial x^{2}}+\frac{\partial^{2} w}{\partial y^{2}}+\frac{\partial^{2} w}{\partial z^{2}}\right)$

Para flujos turbulentos de aplica el modelo Standard $k-\varepsilon$ [31]:

$$
\begin{aligned}
& \frac{\partial}{\partial t}(\rho k)+\frac{\partial}{\partial x_{i}}\left(\rho k u_{i}\right)=\frac{\partial}{\partial x_{j}}\left[\left(\mu+\frac{\mu_{t}}{\sigma_{k}}\right) \frac{\partial k}{\partial x_{j}}\right]+G_{k}+G_{b}-\rho \varepsilon-Y_{m}+S_{k}
\end{aligned}
$$

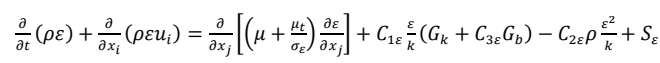

En estas ecuaciones, $G_{k}$ representa la generación de la energía cinética turbulenta debido a los gradientes de velocidad promedio. $\mathrm{G}_{\mathrm{b}}$ es la generación de energía cinética turbulenta debido a la flotación. $Y_{m}$ representa la contribución de la dilatación fluctuante en turbulencia compresible para toda velocidad de disipación. $\mathrm{C}_{1 \varepsilon}, \mathrm{C}_{2 \varepsilon}$ y $\mathrm{C}_{3 \varepsilon}$ son constantes. $\Sigma_{\varepsilon} \mathrm{y} \sigma_{\varepsilon}$ son los números de Prantl turbulentos para $k$ y $\varepsilon$ respectivamente. $S_{k}$ y $S_{\varepsilon}$ son términos fuente definidos por el usuario. La viscosidad turbulenta $\mu_{\mathrm{t}}$ se calcula de la siguiente manera:

$\mu_{t}=\rho C_{\mu} \frac{k^{2}}{\varepsilon}$

son:

Los parámetros de las algunas constantes

$\mathrm{C}_{1 \varepsilon}=1.44, \mathrm{C}_{2 \varepsilon}=1.92, \mathrm{C}_{\mu}=0.09, \sigma_{\mathrm{k}}=1.0, \sigma_{\varepsilon}=1.3$

Para el calcular el coeficiente de arrastre, se usa la ecuación (7), donde $F_{x}$ es la fuerza de arrastre $(\mathrm{N}), \rho$ es la densidad del aire $\left(\mathrm{kg} / \mathrm{m}^{3}\right), \mathrm{v}$ es la velocidad del aire $(\mathrm{m} / \mathrm{s})$ y A es el área frontal del automóvil [1], para este estudio el área es de $4.68 \mathrm{~m}^{2}$.

$C d=\frac{F x}{(1 / 2) \rho v^{2} A}$

La fuerza de sustentación como se percibió desde sus inicios es normal al suelo. A diferencia de la fuerza de arrastre la cual tiene como dirección opuesta a la dirección del vehículo. La ecuación (8) muestra el cálculo de la fuerza de sustentación, donde $\mathrm{F}_{\mathrm{y}}$ es la fuerza de sustentación:

$C l=\frac{F y}{(1 / 2) \rho v^{2} A}$
Para el análisis de este trabajo se usa el aire a bajas velocidades de $35 \mathrm{~m} / \mathrm{s}$ a diferentes números de mallado (859815, 1667308, 4029353, 4491917 y 5313028), la densidad del aire es de $1.2 \mathrm{~kg} / \mathrm{m}^{3}$ y la viscosidad de $1.8 \times 10^{-5}$ Pa.s. Con los resultados de las simulaciones se pretende conocer las fuerzas de arrastre $\mathrm{y}$ sustentación, posteriormente calcular los coeficientes para conocer el mejor modelo aerodinámico con el alerón implementado en la parte trasera que reduzca estas fuerzas en comparación al de los modelos normales.

\section{Resultados}

La Figura 10 muestra los resultados de los coeficientes de arrastre de los diseños normales y con el alerón implementado en la parte trasera para diferentes números de mallado. Con el mallado más fino (5313028 celdas) se obtuvieron un resultado de 0.43 para los diseños normales 1 y 2 . Cuando se les implementa el alerón en la parte trasera, se logró disminuir los coeficientes de arrastre. Con el Diseño 1/Alerón 1 se obtuvo un coeficiente de 0.36, el Diseño 1/Aleron 2 el resultado fue de 0.38 , Diseño 2/Alerón 1 y Diseño 2/Alerón 2 para ambos casos fue de 0.37 .

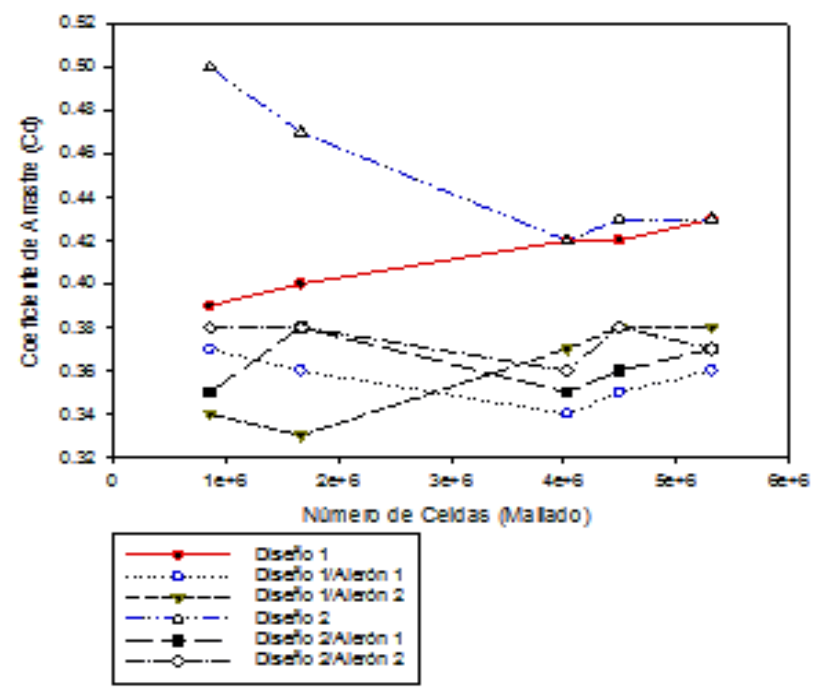

Figura 10 Resultados de los coeficientes de arrastre con diferentes números de celdas en el mallado

Fuente: elaboración propia [Solidworks]

Los resultados de los coeficientes de sustentación son mostrados en la Figura 11. Con el mallado más fino (5313028 celdas) los resultados del diseño 1 y 2 fueron de 0.65 , se observa que con el alerón en la parte trasera se logra disminuir el coeficiente de sustentación para los diseños normales. 
Los modelos Diseño 1/Alerón 1 y Diseño 1/Alerón 2 los resultados fueron de 0.46 y 0.42 , los modelos Diseño 2/Alerón 1 y Diseño 2/Alerón 2 fueron de 0.5 y 0.42 respectivamente. Las Figuras 12 y 13 muestran los resultados de las fuerzas de arrastre y sustentación con diferente número de mallado, se obseva que son el mismo comportamiento como se mostraron anteriormente.

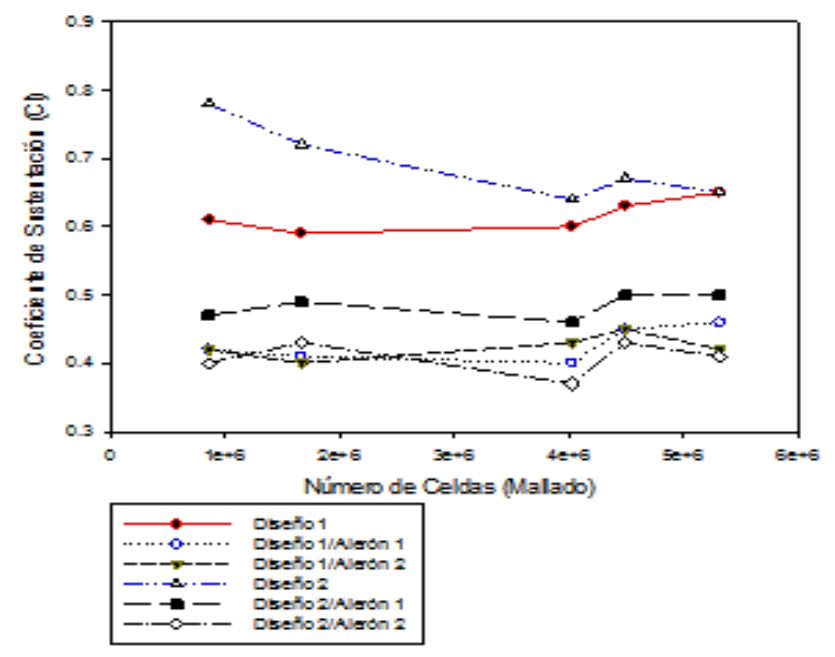

Figura 11 Resultados de los coeficientes de sustentación con diferentes números de celdas en el mallado Fuente: elaboración propia [Solidworks]

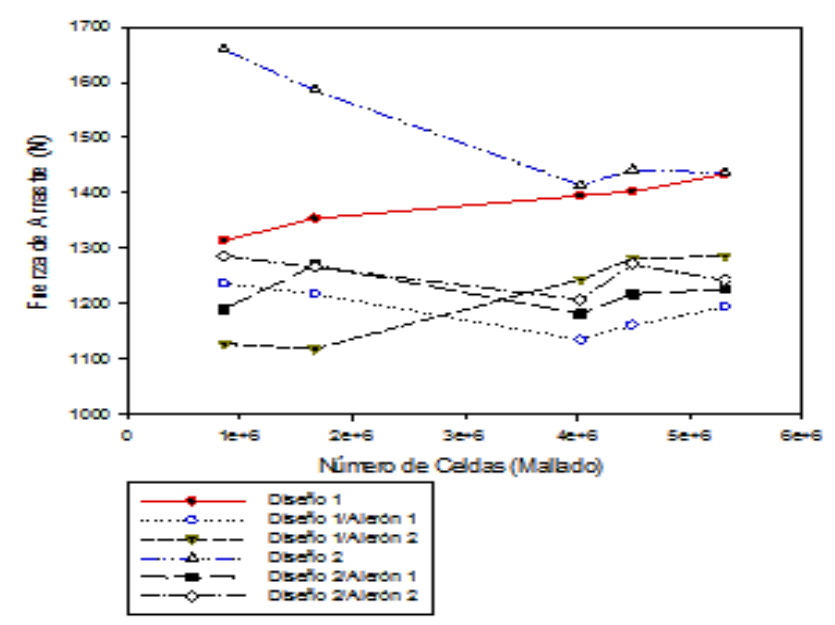

Figura 12 Resultados de las fuerzas de arrastre con diferentes números de celdas en el mallado Fuente: elaboración propia [Solidworks]

Es importante conocer la efectividad del alerón para cada diseño, por eso se realizó un cálculo del porcentaje de reducción de los coeficientes, mientras más alto sea la reducción más efectiva es el alerón.

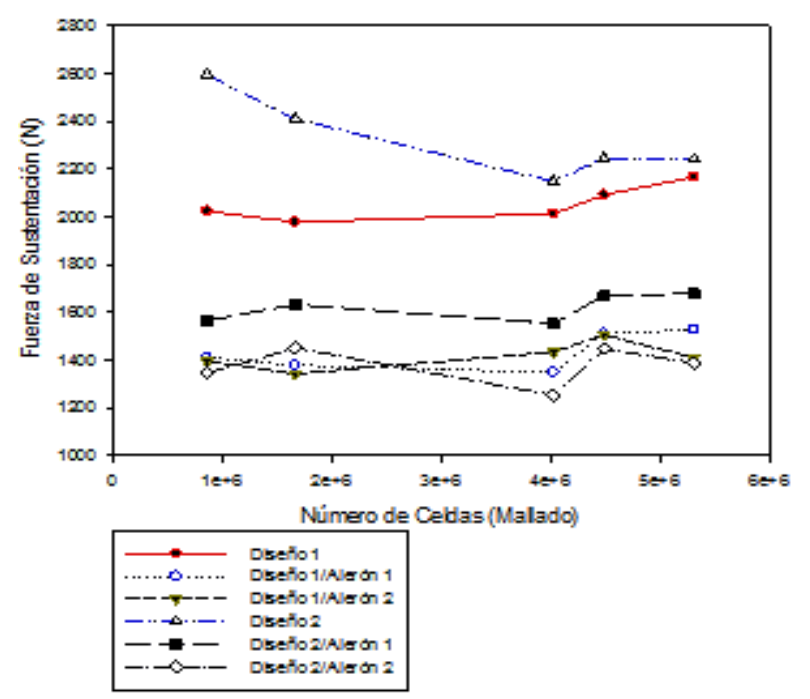

Figura 13 Resultados de las fuerzas de sustentación con diferentes números de celdas en el mallado Fuente: elaboración propia [Solidworks]

La Tabla 1 muestra los porcentajes de reducción de los coeficientes de arrastre del Diseño 1/Alerón 1 y Diseño 2/Alerón 2 en comparación del Diseño 1. El modelo Diseño 1/Alerón 1 tiene una mayor efectividad con un $16.76 \%$.

\begin{tabular}{|r|r|r|}
\hline Diseño 1 & Diseño 1/Alerón 1 & Diseño 1/Alerón 2 \\
\hline 0.43 & 0.36 & 0.38 \\
\hline \%Reducción & 16.76 & 10.34 \\
\hline
\end{tabular}

Tabla 1 Resultados de los porcentajes de reducción de los coeficientes de arrastre del Diseño 1

Fuente: Elaboración propia

La Tabla 2 muestra los resultados de los coeficientes de sustentación de los modelos Diseño 1/Alerón 1 y Diseño 1/Alerón 2 con el porcentaje de reducción. El mayor porcentaje fue de $35 \%$ con el Diseño 1/Alerón 2 y un $29.54 \%$ con el modelo Diseño 1/Alerón 1.

\begin{tabular}{|r|r|r|}
\hline \multicolumn{1}{|c|}{ Diseño 1 } & Diseño 1/Alerón 1 & Diseño 1/Alerón 2 \\
\hline 0.65 & 0.46 & 0.42 \\
\hline \%Reducción & 29.54 & 35 \\
\hline
\end{tabular}

Tabla 2 Resultados de los porcentajes de reducción de los coeficientes de sustentación del Diseño 1

Fuente: elaboración propia

Los resultados de la reducción de los porcentajes del Diseño 2/Aleron 1 y Diseño 2/Alerón 2 en comparación del Diseño 2 son mostrados en las Tablas 3 y 4 . Para el coeficiente de arrastre, la diferencia entre los porcentajes es menor de $14.65 \%$ y $13.53 \%$. En cambio, el mayor porcentaje de reducción del coeficiente de sustentación fue del modelo Diseño 2/Alerón 2 con un 36\%, y el Diseño 2/Alerón 1 fue de $22.54 \%$.

HORTELANO-CAPETILLO, Juan Gregorio, MARTÍNEZVÁZQUEZ, J. Merced y RODRIGUEZ-ORTIZ, Gabriel. Análisis aerodinámico en CFD de los alerones en automóviles convencionales. Revista de Ingeniería Industrial. 2020 


\begin{tabular}{|r|r|r|}
\hline \multicolumn{1}{|c|}{ Diseño 2 } & Diseño 2/Alerón 1 & Diseño 2/Alerón 2 \\
\hline 0.43 & 0.37 & 0.375 \\
\hline \%Reducción & 14.65 & 13.53 \\
\hline
\end{tabular}

Tabla 3 Resultados de los porcentajes de reducción de los coeficientes de arrastre del Diseño 2

Fuente: elaboración propia

\begin{tabular}{|r|r|r|}
\hline \multicolumn{1}{|c|}{ Diseño 2 } & Diseño 2/Alerón 1 & Diseño 2/Alerón 2 \\
\hline 0.65 & 0.5 & 0.42 \\
\hline \%Reducción & 22.54 & 36 \\
\hline
\end{tabular}

Tabla 4 Resultados de los porcentajes de reducción de los coeficientes de sustentación del Diseño 1

Fuente: elaboración propia

Las Figuras 14, 15 y 16 muestran los contornos de velocidad de los modelos Diseño 1, Diseño 1/Alerón 1 y Diseño 1/Alerón 2 de las simulaciones obtenidas con el número de celdas en el mallado de 5313028 a la velocidad de 35 $\mathrm{m} / \mathrm{s}$. Se observa que en la parte trasera del modelo Modelo 1/Aleron 1 y Modelo 1/Alerón 2 forman una estela de vortices con velocidades bajas que hacen que la presión disminuya y el desprendimiento de la capa límite turbulenta se lleve a cabo a una distancia mas lejana, por lo tanto este efecto hace que las fuerzas de arrastre y sustentación disminuyan en comparación del Diseño 1. Cuando mayor sea la presión, mas dificultades tendra en auto para seguir avanzando y retroceda hacia atrás en busca de zonas de menor presión. Por eso es importante el estudio de la capa límite en el diseño para ver donde se llevara a cabo el desprendimiento, con la ayuda del aleron se retraza ese fenómeno.

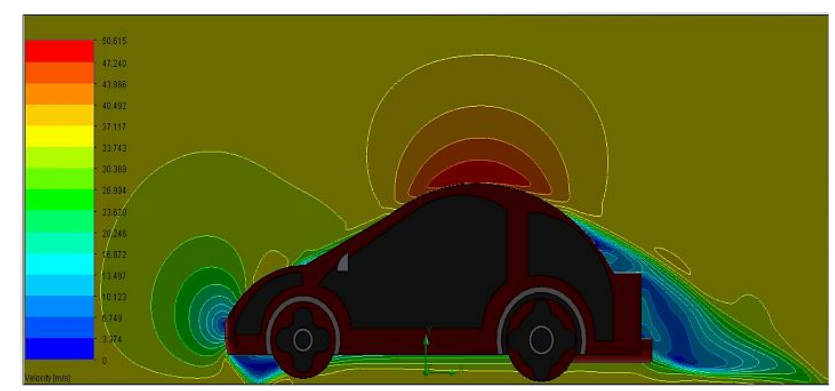

Figura 14 Contornos de velocidad del Diseño 1 Fuente: elaboración propia [Solidworks]

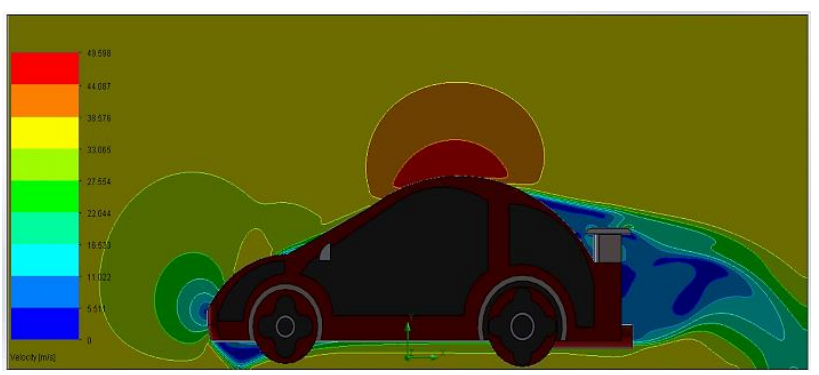

Figura 15 Contornos de velocidad del Diseño 1/Alerón 1 Fuente: elaboración propia [Solidworks]

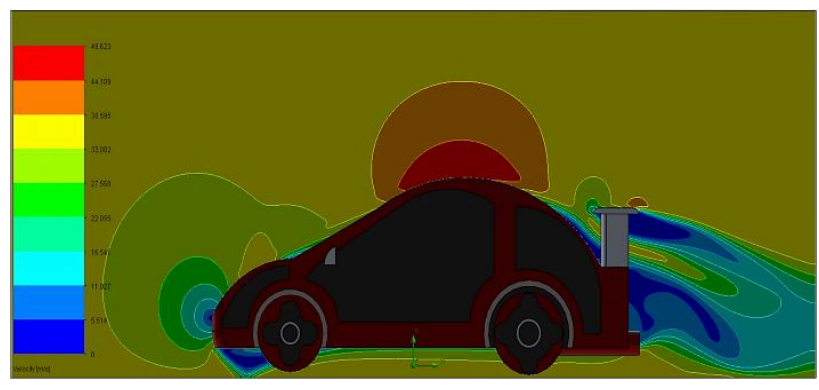

Figura 16 Contornos de velocidad del Diseño 1/Alerón 2 Fuente: elaboración propia [Solidworks]

Los resultados de los contornos de velocidades del Diseño 2, Diseño 2/Alerón 1 y Diseño 2/Alerón 2 y la formación de las estelas en la parte trasera que disminuyen las fuerzas de arrastre y sustentación son mostrados en las Figuras 17,18 y 19 a la velocidad de $35 \mathrm{~m} / \mathrm{s}$ con un número de celdas de 5313028. Comparando el porcentaje de la reducción de los coeficientes de arrastre con el alerón implementado en la parte trasera del Diseño 2, para ambos modelos los porcentajes son muy cercanos, en cambio el coeficiente de sustentación, el modelo Diseño 2/Alerón 2 se logró reducir este coeficiente hasta un $36 \%$.

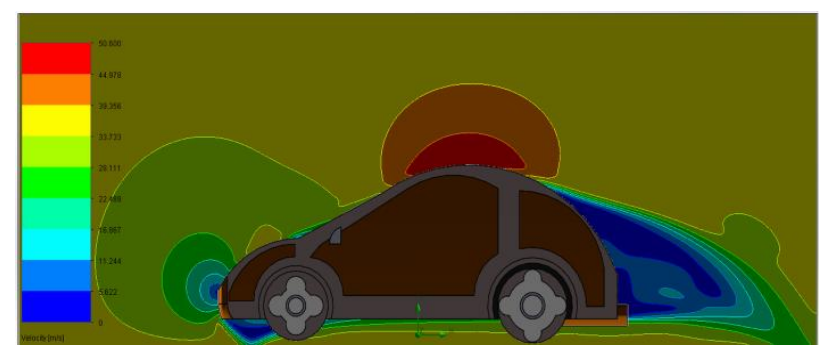

Figura 17 Contornos de velocidad del Diseño 2 Fuente: elaboración propia [Solidworks]

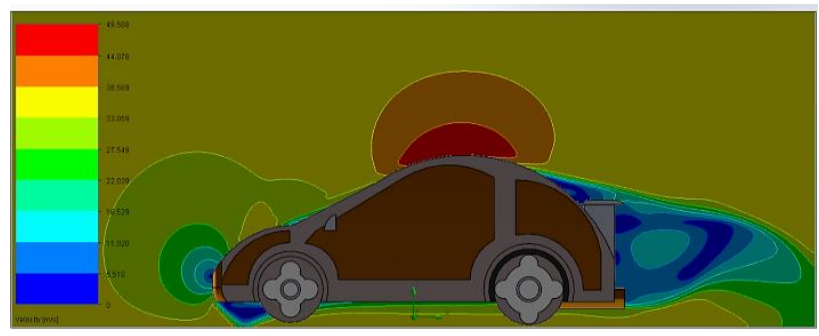

Figura 18 Contornos de velocidad del Diseño 2/Alerón 1 Fuente: elaboración propia [Solidworks]

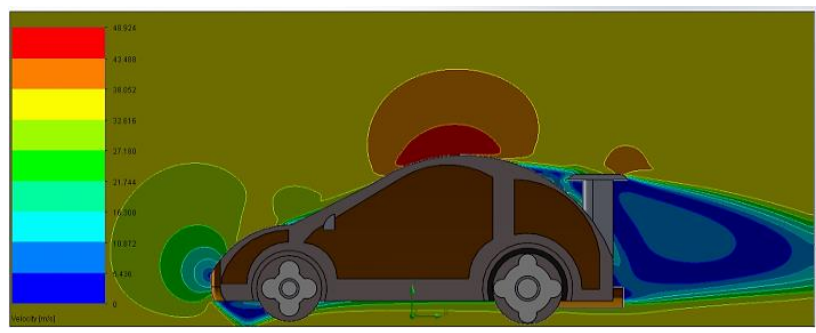

Figura 19 Contornos de velocidad del Diseño 2/Alerón 2 Fuente: elaboración propia [Solidworks]

HORTELANO-CAPETILLO, Juan Gregorio, MARTÍNEZVÁZQUEZ, J. Merced y RODRIGUEZ-ORTIZ, Gabriel. Análisis aerodinámico en CFD de los alerones en automóviles convencionales. Revista de Ingeniería Industrial. 2020 
Las Figuras 20, 21, 22, 23 y 24 muestran las formaciones de vórtices de baja velocidad en el rango de $0-5 \mathrm{~m} / \mathrm{s}$ en la parte trasera para cada modelo que hacen que la presión disminuya y el desprendimiento de la capa límite turbulenta se lleve a cabo a una distancia mas lejana.

El Diseño 1/Aleron 1 mostró mejores resultados en la reducción de los coeficientes de arrastre y el Diseño 1/Aleron 2 mostró mejores resultados en la sustentación.

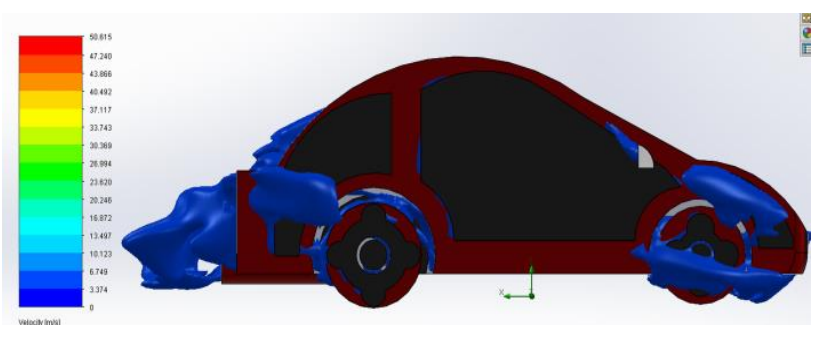

Figura 20 Contornos de baja velocidad del Diseño 1 Fuente: elaboración propia [Solidworks]

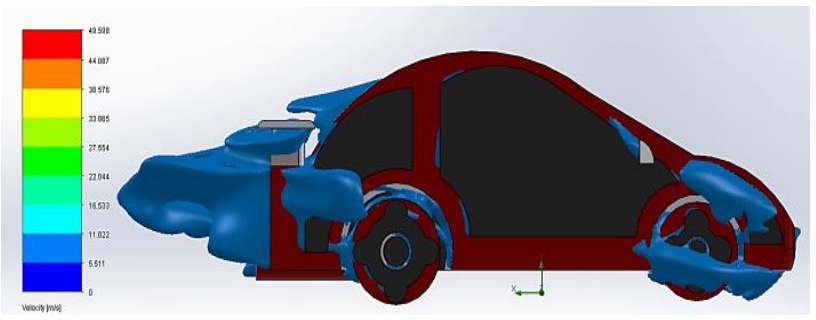

Figura 21 Contornos de baja velocidad del Diseño 1/Alerón 1

Fuente: elaboración propia [Solidworks]

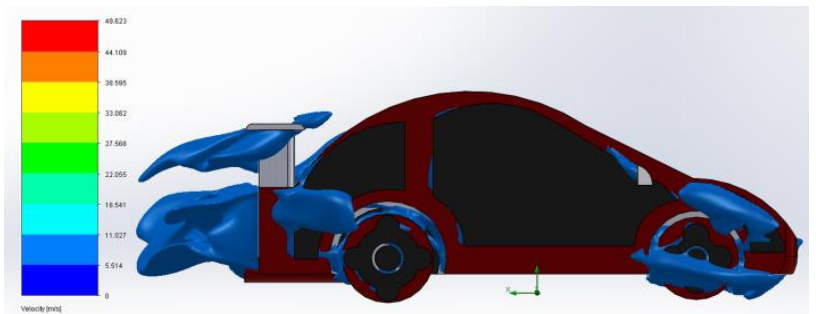

Figura 22 Contornos de baja velocidad del Diseño 1/Alerón 2

Fuente: Elaboración propia [Solidworks]

El Diseño 2/Alerón 2 mostró mejores resultados para reducir las fuerzas en la sustentación y en el arrastre en comparación del Diseño 2/Alerón 1. La altura del aleron favorecio al diseño de la parte trasera como se aprecia en los vortices mostrados en esta Figura 25.

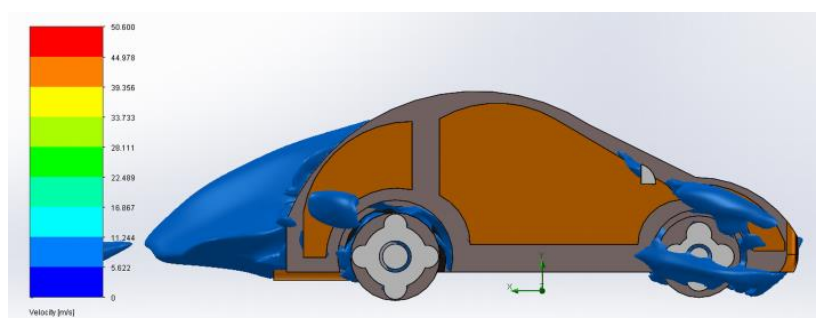

Figura 23 Contornos de baja velocidad del Diseño 2 Fuente: elaboración propia [Solidworks]

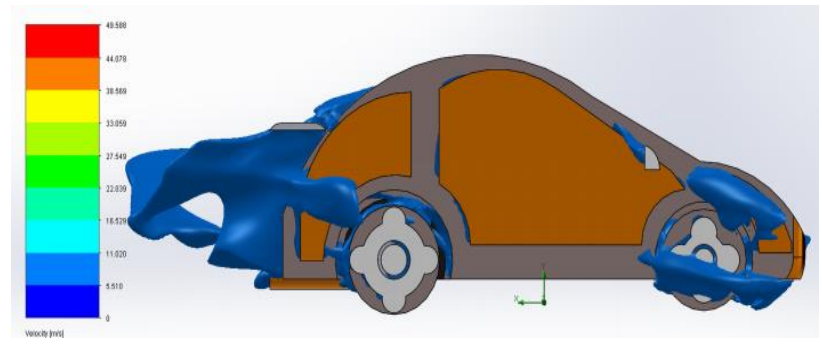

Figura 24 Contornos de baja velocidad del Diseño 2/Alerón 1

Fuente: elaboración propia [Solidworks]

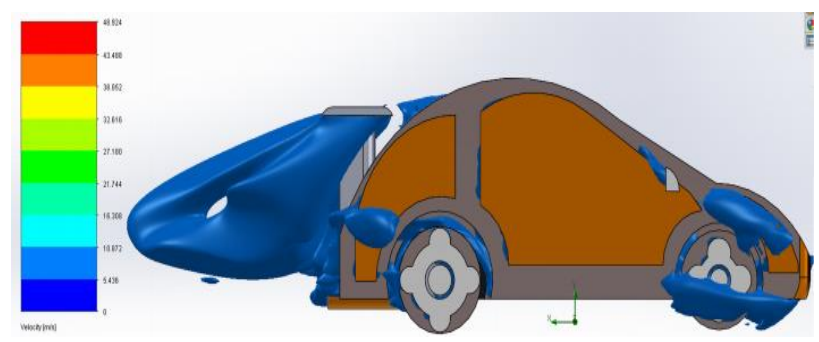

Figura 25 Contornos de baja velocidad del Diseño 2/Alerón 2

Fuente: elaboración propia [Solidworks]

Las Figuras 26 y 27 muestran la comparación de contornos de velocidad a $20 \mathrm{~m} / \mathrm{s}$ de los modelos Diseño 1/Alerón 2 y Diseño 2/Alerón 2.

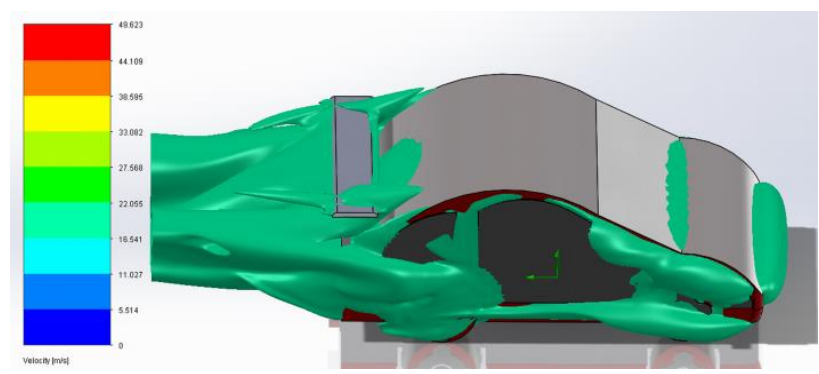

Figura 26 Contornos de velocidad a $20 \mathrm{~m} / \mathrm{s}$ del Diseño 1/Alerón 2

Fuente: elaboración propia [Solidworks] 

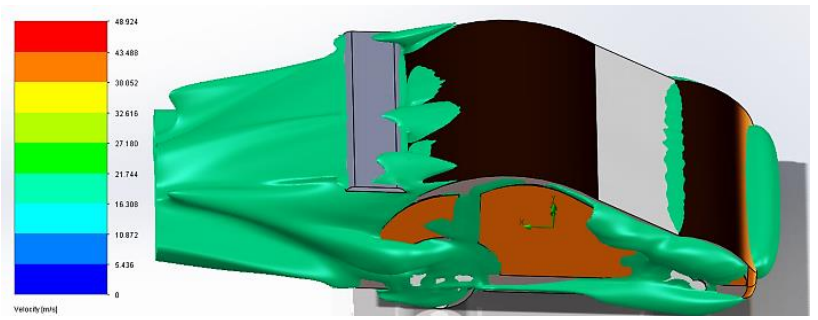

Figura 27 Contornos velocidad a $20 \mathrm{~m} / \mathrm{s}$ del Diseño 2/Alerón 2

Fuente: elaboración propia [Solidworks]

Las Figuras 28 y 29 muestran los resultados de las simulaciones obtenidas con el número de celdas de 5313028 para los Diseños 1 y 2 , donde se muestran los contornos de presión en la superficie del automóvil que ejerce el aire a la velocidad de $35 \mathrm{~m} / \mathrm{s}$. Se observa que en la parte del frente del auto existe la mayor presión de $102353 \mathrm{~Pa}$ en promedio para todas las simulaciones y en la parte superior muestra presiones bajas a $101325 \mathrm{~Pa}$.

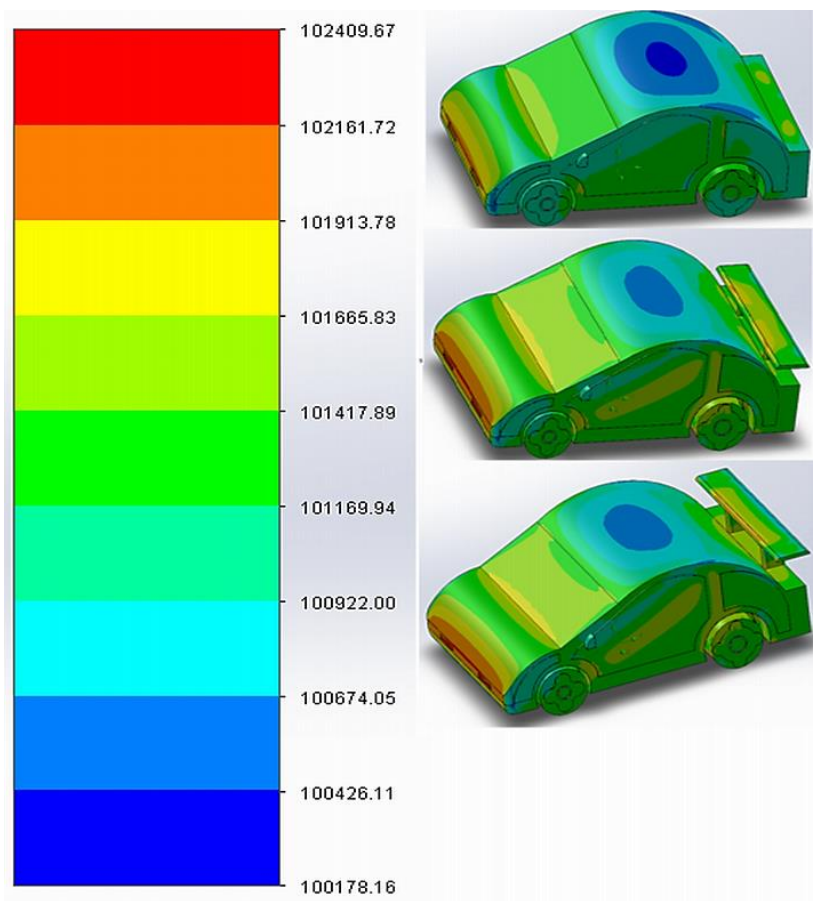

Pressure $[\mathrm{Pa}]$

Surface Plot 1: contours

Figura 28 Contornos de presión de los modelos del Diseño 1

Fuente: elaboración propia [Solidworks]

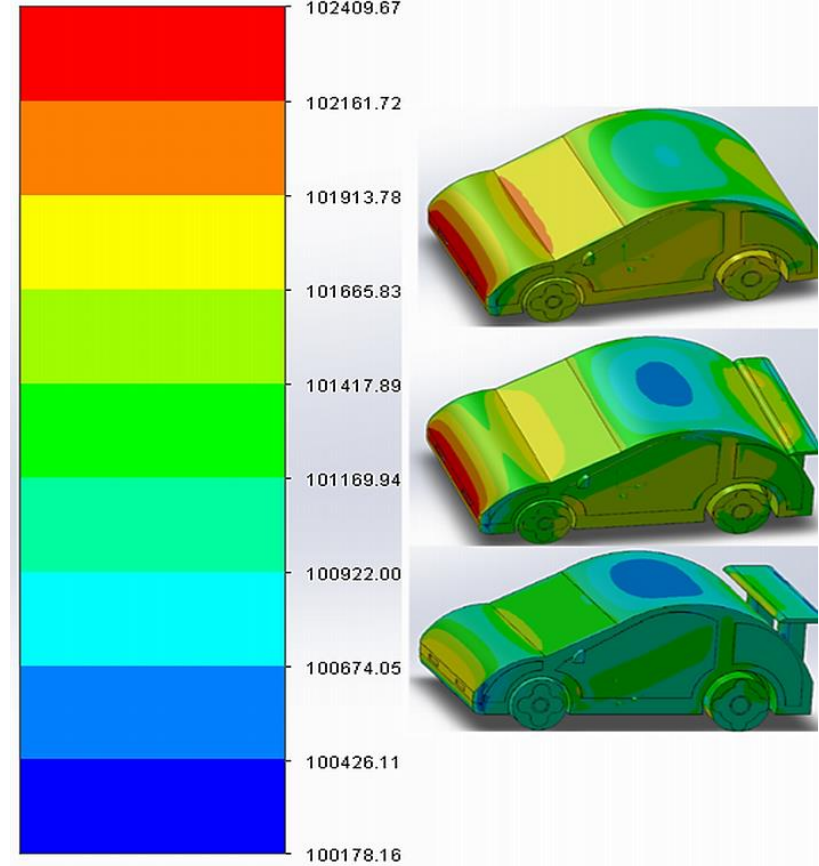

Pressure $[\mathrm{Pa}]$

Surface Plot 1: contours

Figura 29 Contornos de presión de los modelos del Diseño 2

Fuente: elaboración propia [Solidworks]

\section{Conclusiones}

Realizando una comparación entre los 2 diseños de alerones aplicados en los 2 modelos con diferente diseño en la parte trasera, efectivamente se logra disminuir los coeficientes de arrastre y sustentación en comparación con el diseño normal. Para un automóvil convencional que tenga un diseño similar al del modelo 1 , se recomienda que el alerón se coloque a una altura a $45 \mathrm{~cm}$ aproximadamente para que sea más efectivo en reducir las fuerzas. En cambio, los automóviles que tengan un diseño similar al del modelo 2 , se recomienda que el alerón tenga una altura de $45 \mathrm{~cm}$ aproximadamente para mayor efectividad. Podemos concluir que el implemento de un alerón en un auto normal garantiza la reducción de los coeficientes de arrastre y sustentación, mayor estabilidad del automóvil al momento girar una curva o en un trayecto normal en el camino. Por lo tanto, estos coeficientes son afectados por el diseño y dimensiones del automóvil. Por parte del equipo de cómputo, mientras el mallado sea más fino, más tiempo se tardará en resolver la simulación y los resultados serán más exactos a la realidad dependiendo de las características de la computadora. 


\section{Referencias}

[1] Introduction to Fluid Mechanics, Sixth Edition, (2003). Robert W. Fox, Alan T. MacDonald, Philip J. Pritchard.

[2] J. Kelly (2004). "American Cars", 19601972. Every Model, Year by Year. McFlarland. p. 97. ISBN: 9780786412730.

[3] F. Milliken, L. Milliken, Race Car Vehicle Dynamics, Twelfth printing, SAE, USA.

[4] Hall J. "What's Jim Hall Really Like?" Automobile Quarterly, VIII, 3, Spring 1970. pp. 282-293.

[5] R. B. Sharma, Ram Bansal. "CFD Simulation for Flow over Passenger Car Using Tail Plates for Aerodynamic Drag Reduction, IOSR Journal of Mechanical and Civil Engineering (IOSR JMCE). ISBN: 2320-334X. 75 (2013) 28-35.

[6] Carr G. W. "The Aerodynamics of Basic Shapes for Road Vehicles. Part 3: Streamlined Bodies" The Motor Industry Research Association, Warwickshire, England, Report No. 107/4, 1969.

[7] Goetz H. "The Influence of Wind Tunnel Test on Body Design, Ventilation and Surface Deposits of Sedans and Sports Cars" SAE paper No. 710212, 1971.

[8] Liebeck R. H. "Design of Subsonic Airfoils Systems" AIAA Journal of Aircraft, 15, 9, September 1978, pp. 547-561.

[9] R. H. Barnard, H. Vaughan. "The Aerodynamic Tuning of a group C Sports Racing Car" Journal of Wind Engineering and Industrial Aerodynamics, 22 (1986) 279-289.

[10] ChienHsiung Tsai, Lung Ming Fu, Chang Hsien Tai, Yen Loung Huang, Jik Chang Leong. "Computational aero-acoustic analysis of a passenger car with a rear spoiler" Applied Mathematical Modelling. 33 (2009) 3661-3673.
[11] Xingjun Hu, Rui Zhang, Jian Ye, Xu Yan, Zhiming Zhao. "Influence of Different Diffuser Angle on Sedan's Aerodynamic Characteristics". Physics Procedia 22 (2011) 239-245.

[12] S. M. Rakibul Hassan, Toukir Islam, Mohammad Ali, Md. Quamrul Islam. "Numerical Study on Aerodynamic Drag Reduction of Racing Cars". Procedia Engineering 90 (2014) 308-313.

[13] Rubel Chandra Das, Mahmud Riyad, " $C F D$ Analysis of Passenger Vehicleat Various Angle of Rear End Spoiler". Procedia Engineering 194 (2017) 160-165.

[14] Krzysztof Kurec, Michal Remer, Tobiasz Mayer, Sylwester Tudruj, Janusz Piechna. "Flow control for a car-mounted rear wing". International journal of Mechanical Sciences. Volume 152 (2019) 384-399.

[15] Gilhaus, R. Hoffmann. "Directional Stability, Aerodynamic od Road Vehicles, in: W.H. Hucho (Ed), SAE International, Warrendale. PA, 1998.

[16] J.R. Callister, A.R. George, Wind Noise, Aerodynamics of Road Vehicles, in: W.H. Hucho (Ed.), SAE International, Warrendale, PA, 1998.

[17] F.R. Bailey, H.D. Simon, Future Directions in Computing and CFD, AIAA Paper 922734, 1992.

[18] H. Taeyoung, V. Sumantran, C. Harris, T. Kuzmanov, M. Huebler, T. Zak, Flow-field simulations of three simplified vehicle shapes and comparisons with experimental measurements, SAE Transactions 106 (1996) 820835.

[19] Cooper K. R. Commercial vehicle aerodynamic drag reduction: historical perspective as a guide. In: The Aerodynamics of Heavy Vehicles: Trucks, Buses and Trains. Springer, Berlin. Heidelberg, pp. 9-28. 2004.

[20] Sovran G. Tractive-energy-based Formulae for the Impact of Aerodynamics on Fuel Economy Over the EPA Driving Schedules, (No. 830304) SAE Technical Paper. 
[21] Katz J. Aerodynamics of race cars. Annu. Rev. Fluid Mech. 38. 27-63. 2006.

[22] Regert T, Lajos T. Description of flow field in the wheelhouses of cars. International Journal Fluid Flow. 28 (4). 616-629. 2007.

[23] Wong D. M, Mair W. A, Boat-tailed afterbodies of square section as dragreduction devices. J. Wind Eng. Ind. Aerod. (1983) 12 (2), 229-235.

[24] Lee E. J, Lee S. J, Drag reduction of a heavy vehicle using a modified boat tail with lower inclined air deflector. J. Visual. 20 (4), 743752. 2017.

[25] Mariotti A, Buresti G, Gaggini G. Salvetti M. V, Separation control and drag reduction for boat-tailed axisymmetric bodies through contoured transverse grooves. J. Fluid Mech. 832, 514-549. 2017.

[26] Beaudoin J. F, Aider J. L, Drag and lift reduction of a $3 D$ bluff body using flaps. Exp. Fluid. 44 (4), 491. 2008.

[27] Fourrié G, Keirsbulck L, Labraga L, Gilliéron P, Bluff-body drag reduction using a deflector. Exp. Fluid. 50 (2), 385-395. 2011.

[28] Hanfeng W, Yu Z, Chao Z, Xuhui H, Aerodynamic drag reduction of an Ahmed body based on deflectors. J. Wind Eng. Ind. Aerod. 148, 34-44. 2016.

[29] Grandemange M, Gohlke M, Cadot O, Effect on drag of the flow orientation at the base separation of a simplified blunt road vehicle. Exp. Fluid. 54 (5). 1529. 2013.

[30] Urquhart M, Sebben S, Sterken L, Numerical analysis of a vehicle wake with tapered rear extensions under yaw conditions. J. Wind Eng. Ind. Aerod. 179, 308-318. 2018.

[31] V. Yakhot and L. M. Smith. The renormalization group, the E-expansion and derivation of turbulence models. Journal of Scientific Computing, Vol. 7, no. 1, pp. 35-61, 1992. 